\section{Targeting TB persistence}

\section{By Lauren Martz, Staff Writer}

A group of researchers from California and New York has identified a small molecule with activity against currently intractable nonreplicating stages and drug-resistant strains of Mycobacterium tuberculosis. ${ }^{1}$ The compound, which works by blocking a cell wall biosynthesis enzyme and a cofactor biosynthesis enzyme, could be combined with existing tuberculosis drugs to help shorten treatment duration and prevent or eliminate the emergence of resistant bacteria.

One of the major challenges of treating TB is bacterial persistence, which occurs when the bacteria enter a dormant, nonreplicating phase. Most marketed drugs are not effective against dormant $\mathrm{TB}$, and new compounds are still mostly screened against growing bacteria, which have different metabolic properties and requirements than latent bacteria and only represent a fraction of the life cycle of $M$. tuberculosis.

As a result, the duration of standard care administration regimes spans from several months to years, leading to poor compliance and the emergence of resistant strains.

To identify new therapeutics for TB, Peter Schultz, William Jacobs, Feng Wang and colleagues created a Mycobacterium biofilm formation screen. The team hypothesized that measuring bacterial growth as a biofilm might mimic in vivo conditions during bacterial infection better than other cell culture models and could help identify molecules with new mechanisms of action.

Schultz is professor of chemistry at The Scripps Research Institute and founding director of the California Institute for Biomedical Research (Calibr). Jacobs is professor in the Department of Microbiology and Immunology and the Department of Genetics at the Albert Einstein College of Medicine of Yeshiva University. Wang is a principal investigator at Calibr.

The screen of 70,000 molecules used M. smegmatis, a nonpathogenic bacterium that is more suitable for preclinical screening than M. tuberculosis. Bacterial biofilm growth assays testing the most promising hits showed that TCA1 potently and selectively inhibited Mycobacterium-genus bacteria over other pathogenic bacteria.

In bacterial killing assays using drug-sensitive M. tuberculosis, TCA1 had comparable efficacy to the generic TB drugs isoniazid and rifampicin, and a combination of TCA1 and either drug eradicated the bacteria. In assays using multidrug-resistant strains of the bacteria, TCA1 was more effective than isoniazid, rifampicin or no treatment, and the combination of TCA1 and isoniazid eliminated a multidrugresistant strain.

TCA1 also was effective against extensively drug-resistant M. tuberculosis, suggesting it has a different mechanism of action than currently available drugs.

Moreover, TCA1 was effective in a nutrient-starvation $M$. tuberculosis assay, which measures the effect of therapeutics against bacteria in a dormant, nonreplicating state.

In mouse models for chronic and acute M. tuberculosis infection, oral treatment with TCA1 decreased bacterial burden compared with no treatment and was as effective as treatment with isoniazid and rifampicin. TCA1 did not cause weight loss or other adverse effects.

Genetic and affinity-binding studies identified TCA1's targets as M. tuberculosis decaprenylphosphoryl- $\beta$-D-ribose 2 '-oxidase (dprE1) and M. tuberculosis molybdopterin biosynthesis protein (moeW). dprE1 is involved in cell wall biosynthesis, and moeW plays a role in molybdenum cofactor biosynthesis.

The team also found evidence that dprE1 inhibition contributes to bactericidal activity in replicating bacteria, and moeW inhibition may contribute to bactericidal activity in latent bacteria.

Data were published in the Proceedings of the National Academy of Sciences, and the team included researchers from the University of Birmingham, Comenius University in Bratislava, the Swiss Federal Institute of Technology Lausanne, the Global Alliance for TB Drug Development and the University of Illinois at Chicago.

"TCA1 has a relatively unique mechanism of action that appears to function differently from all previous antimicrobials for tuberculosis examined, making it quite an exciting compound," said Jeffrey Cirillo, professor in the Department of Microbial and Molecular Pathogenesis at the Texas A\&M Health Science Center College of Medicine. "The great benefits of TCA1 appear to be its high potency under nearly any conditions, including where bacteria are not replicating, which is a condition where very few drugs currently work."

\section{Analog data}

Calibr has partnered with the TB Alliance to develop TCA1 and related molecules.

Schultz told SciBX that his group has developed significantly more potent analogs of TCA1. "We have reasonable pharmacokinetics for oral dosing of some analogs," he said.

"Being from a novel class, these compounds are expected to help reduce and combat drug resistance, as they should be effective in treating both drug-sensitive tuberculosis and drug-resistant tuberculosis," said Takushi Kaneko, senior research fellow at the TB alliance. 


\section{ANALYSIS}

\section{TARGETS \& MECHANISMS}

\section{"Being from a novel class, these compounds are expected to help reduce and combat drug resistance, as they should be effective in treating both drug-sensitive tuberculosis and drug- resistant tuberculosis." \\ - Takushi Kaneko, \\ Global Alliance for TB Drug Development}

He added that "killing nonreplicating $M$. tuberculosis cells is considered to be the most critical in shortening tuberculosis treatment duration."

According to Schultz, it is too early to predict how much TCA1 or related molecules could decrease treatment time.

"It is usually difficult to project the treatment time in vivo according to the in vitro activities. For M. tuberculosis, it is even more difficult due to the lack of good animal models for latent infection. However, based on the performance of known tuberculosis drugs, those drugs that have good activity against nonreplicating $M$. tuberculosis in vitro do have potential to shorten treatment time in humans," he said.

Schultz added that in addition to killing multidrug-resistant and extensively drug-resistant $M$. tuberculosis strains, his team has found that TCA 1 shows a very low frequency of inducing resistance mutations. "This may be related to the dual mechanisms of action of this compound," he said.
Schultz said the work has been patented by Calibr and the IP is available for licensing.

Martz, L. SciBX 6(26); doi:10.1038/scibx.2013.646

Published online July 11, 2013

\section{REFERENCES}

1. Wang, F. et al. Proc. Natl. Acad. Sci. USA; published online June 17, 2013; doi:10.1073/pnas.1309171110

Contact: Peter G. Schultz, The Scripps Research Institute, La Jolla, Calif.

e-mail: schultz@scripps.edu

Contact: William R. Jacobs Jr., Albert Einstein College of Medicine of Yeshiva University, Bronx, N.Y.

e-mail: jacobsw@hhmi.org

\section{COMPANIES AND INSTITUTIONS MENTIONED}

Albert Einstein College of Medicine of Yeshiva University, Bronx, N.Y.

California Institute for Biomedical Research, La Jolla, Calif. Comenius University in Bratislava, Bratislava, Slovakia Global Alliance for TB Drug Development, New York, N.Y. The Scripps Research Institute, La Jolla, Calif.

Swiss Federal Institute of Technology Lausanne, Lausanne, Switzerland

Texas A\&M Health Science Center College of Medicine, Bryan, Texas

University of Birmingham, Birmingham, U.K. University of Illinois at Chicago, Chicago, III. 